TRANS · núm. $24 \cdot 2020$

DOSIER $\cdot 129-144$

Este artículo investiga la audiodescripción en Televisión Española (TVE) en su emisión en TDT y online. Esta modalidad de traducción intersemiótica está recogida en la normativa europea y española como un servicio de accesibilidad para personas con discapacidad visual orientado al cumplimiento de derechos fundamentales como la igualdad en el acceso a los contenidos mediáticos. Mediante un análisis de contenido y un estudio de recepción basado en encuestas a usuarios, el estudio describe los niveles de audiodescripción de la televisión pública y mide la satisfacción que los receptores tienen de este servicio.

PALABRAS CLAVE: traducción audiovisual, accesibilidad, audiodescripción, televisión, personas ciegas, discapacidad visual.

\title{
La audiodescripción en televisión lineal y bajo demanda: el caso de TVE
}

\author{
Victoria García-Prieto \\ Universidad de Sevilla
}

\section{Audio Description on Linear and On-Demand Television: the case of TVE}

This article studies the audio description of Television Española (TVE) in its broadcast on DTT and online. This modality of inter-semiotic translation is included in the European and Spanish regulations as an accessibility service for people with sight loss aimed at complying with fundamental rights such as equal access to media content. Through a content analysis and a reception study based on user surveys, the study describes the levels of audio description of public television and measures the satisfaction of receivers with this service.

KEY WORDS: audio visual translation, accessibility, audio description, television, blind people, sight loss. 


\section{1. INTRODUCCIÓN}

Este artículo investiga la audiodescripción en Televisión Española (TVE) como servicio de accesibilidad para personas con discapacidad visual. En concreto, analiza los niveles de audiodescripción en televisión lineal y online, ya sea en directo o bajo demanda, y los compara con los niveles mínimos exigidos por ley para establecer si la televisión pública cumple o no la normativa. Asimismo, recopila la opinión de las personas ciegas que utilizan este servicio mediante encuestas para determinar su nivel de satisfacción con la audiodescripción de TVE.

La audiodescripción es una modalidad de traducción intersemiótica de lenguaje visual no verbal a lenguaje hablado (Jiménez Hurtado y Soler Gallego, 2015; Snyder, 2005). Se utiliza para hacer que el teatro, las películas, los programas de televisión y otros entornos sean accesibles para personas con discapacidad visual: «An additional narration describes the action, body language, facial expressions, scenery and costumes. The description fits in between the dialogue and does not interfere with important sound and music effects» (Benecke, 2004: 78).

En general, se puede afirmar que cuanta mayor es la pérdida visual, mayor es la necesidad de este servicio (Lopez et al., 2018). Sin embargo, no debemos olvidar que se dirige a un grupo muy heterogéneo. Así, no todas las personas con discapacidad visual son ciegas, muchas tienen restos visuales y muchas han tenido visión en algún momento de su vida. Del mismo modo, puede haber personas jóvenes con distintos tipos de pérdida visual que se beneficien de la audiodescripción en televisión, pero también personas mayores, dada la común pérdida de audición y visión que se produce con la edad (Fryer, 2016).

Además, la audiodescripción es un servicio útil en los procesos de aprendizaje. Por ejem- plo, los programas infantiles audiodescritos son prácticos para el aprendizaje de la lengua (Palomo López, 2010) y, por ello, es necesario prestar especial atención a la audiodescripción de estos contenidos. En este sentido, Palomo López defiende que la audiodescripción para niños y niñas sea considerada como un tipo diferente, con sus características y requisitos particulares: «Visually impaired children have needs different from the general public, as they are more likely to have delayed language than other children because of the gaps in their experience» (Palomo López, 2010: 213). Asimismo, en el caso de las personas con dificultades de aprendizaje, la audiodescripción facilita el seguimiento del argumento y la narrativa de diversos contenidos audiovisuales, aunque generalmente no es suficiente para lograr un entendimiento total del contenido (Franco et al., 2015).

En resumen, la audiodescripción comprende la descripción hablada de aquellos elementos visuales que son relevantes para el seguimiento de los programas de televisión y está orientada a hacer que los contenidos audiovisuales sean accesibles para personas ciegas o con baja visión. Según Fryer (2016), se debe audiodescribir quién está en escena, dónde está ubicado, qué está haciendo y cómo lo hace. Sin embargo, la información que se incluye en la audiodescripción depende directamente de las propias características del programa y del tipo de contenido del que se trate, por lo que la decisión final depende en buena medida de la persona que realiza la descripción (Vercauteren, 2007).

Teniendo en cuenta su importancia como servicio de accesibilidad, la audiodescripción ha sido incluida explícitamente en la normativa audiovisual europea y española. En Europa, después de diversas iniciativas relacionadas con la igualdad y los derechos de las personas con discapacidad, de entre las que destaca el Año 
Europeo de las Personas con Discapacidad en 2003 (Díaz-Cintas y Remael, 2014), la Directiva 2010/13/UE de Servicios de Comunicación Audiovisual incluyó una mención expresa al subtitulado, la audiodescripción y la lengua de signos como servicios que los operadores de televisión tenían la obligación de ofrecer para hacer sus contenidos accesibles a personas con discapacidad visual o auditiva.

También la Ley 7/2010 General de la Comunicación Audiovisual en España menciona la obligatoriedad de incluir estos servicios de accesibilidad y establece unos valores mínimos que los canales de televisión públicos y privados deben cumplir. En el caso que nos ocupa en este artículo, la ley establece un mínimo de 10 horas semanales con audiodescripción para los canales de televisión públicos. Sin embargo, esta norma no establece el horario en el que deben emitirse los contenidos accesibles ni el tipo de programas que deben incluir los servicios de accesibilidad - subtitulado, audiodescripción y lengua de signos- Además, se ciñe exclusivamente a la TDT, por lo que tampoco incluye la obligación de hacer accesibles los contenidos emitidos en canales de pago, plataformas de vídeo bajo demanda o contenidos online.

Tomando este marco legal como punto de partida, nuestra investigación estudia si la audiodescripción de TVE alcanza el mínimo de 10 horas semanales exigidas por ley, pero también abarca el resto de aspectos mencionados que no aparecen en la normativa audiovisual española y que, por lo tanto, quedan a juicio de la propia televisión. Asimismo, recopila y expone el nivel de satisfacción que las personas ciegas usuarias tienen sobre todos estos parámetros de la audiodescripción de TVE.

La introducción de la audiodescripción - junto con el subtitulado y la lengua de signos-en la normativa nacional e internacional se debe a la importancia del acceso a los medios de comunicación para la inclusión y la participación de toda la ciudadanía en las sociedades democráticas. Así se expone en la Convención internacional sobre los derechos de las personas con discapacidad, aprobada por Naciones Unidas en 2006 y ratificada por España en 2008. Del mismo modo, la citada Directiva 2010/13/UE de Servicios de Comunicación Audiovisual expone así la vinculación entre la accesibilidad audiovisual y la participación social:

El derecho de las personas con discapacidad y de las personas de edad avanzada a participar e integrarse en la vida social y cultural de la comunidad está vinculado indisolublemente a la prestación de unos servicios de comunicación audiovisual accesibles. La accesibilidad de los servicios de comunicación audiovisual incluye, sin limitarse a ellos, aspectos como el lenguaje de signos, el subtitulado, la descripción acústica y menús de pantalla fácilmente comprensibles. (Directiva 2010/13/UE: 95/6)

Efectivamente, numerosos investigadores en la materia han puesto de manifiesto la importancia de que los medios de comunicación sean accesibles para lograr la inclusión social de las personas con discapacidad (Bachmeier, 2014; Molina Saorín, 2017; Storch de Gracia y Asensio, 2007). Kurz y Mikulasek (2004) defienden que la televisión debe ser accesible, ya que juega un papel lingüístico, educacional, político y social. En este sentido, Rimmerman (2012) no duda en calificar de «extremadamente importante» el papel que juegan los medios de comunicación en la exclusión o inclusión social de las personas con discapacidad.

The media leads popular culture, stereotypes, language and social trends, and, as such, depicts and also underrepresents people with disabilities. The impression is that in recent years there is a 
recognition that the media has to present a more balanced and progressive image of people with disabilities. (Rimmerman, 2012: 180)

Este autor habla de las políticas que promueven la inclusión social y menciona explícitamente las leyes que impulsan la accesibilidad audiovisual para personas sordas a través del subtitulado y la lengua de signos, cuestión lógicamente extensible al caso de la audiodescripción para las personas con discapacidad visual. Por lo tanto, como se ha expuesto, este trabajo se justifica no solo por su interés científico, sino también por su carácter social y por su defensa del derecho de las personas con discapacidad a acceder al contenido audiovisual.

Además, actualmente, el contenido audiovisual no se ciñe exclusivamente a la televisión lineal, sino que también se consume online de múltiples formas. De hecho, el consumo audiovisual en línea no ha dejado de crecer en los últimos años y, según el Panel de Hogares de la Comisión Nacional de los Mercados y la Competencia (CNMC), el 37,1\% de los hogares en España consumen contenidos en línea en 2019 (CNMC, 2019). Por ello, este trabajo no analiza solo la televisión tradicional, sino que también estudia los niveles de audiodescripción de los contenidos de TVE online emitidos en su página web, ya sea en directo o a la carta.

Por último, es necesario justificar también la aportación de este trabajo en términos de pluralismo y diversidad de los medios de comunicación. En este sentido, la Comisión Europea establece hasta cinco formas distintas de pluralismo mediático, de entre las que destaca, para este estudio, el pluralismo cultural. Este tipo de pluralismo hace referencia a la necesidad de que los medios de comunicación sean accesibles y representen adecuadamente a toda la diversidad social, incluyendo a las personas con discapacidad
(Labio Bernal, 2014). De esta forma, la Comisión Europea define el pluralismo cultural en los medios de comunicación con las siguientes palabras:

Cultural pluralism in the media refers to the fair and diverse representation of and expression by (i.e. passive and active access) the various cultural and social groups, including ethnic, linguistic, national and religious minorities, disabled people, women and sexual minorities, in the media. It comprises a plurality of themes and voices being present in the media, socialisation through multiple forms of media access and participation, choice between different forms of interaction and the representation of diverse values, viewpoints and roles, in which citizens belonging to various cultural and social groups, including national, ethnic, and linguistic groups, women, disabled people and sexual minorities, can recognise themselves. (Comisión Europea, 2009: 12)

Para terminar, es necesario contextualizar el origen y la evolución de la audiodescripción en TVE hasta el momento de nuestro estudio. Aunque la incorporación de la audiodescripción en televisión comenzó a ser obligatoria en España con la aprobación de la ley audiovisual de 2010, TVE ya había implantado este servicio un año antes, en 2009 (CMT, 2010), pero no fue hasta 2015 cuando la audiodescripción comenzó a experimentar un crecimiento significativo. Como se aprecia en el siguiente gráfico, los niveles siguieron creciendo en 2016, pero se mantuvieron similares en 2017 (este es el último año del que aporta información TVE y coincide con el inicio de nuestro análisis propio). Tomando como partida la justificación del estudio expuesta y el contexto regulatorio actual, el objetivo principal de este artículo es evaluar la accesibilidad de los contenidos de TVE para personas con discapacidad visual a través de la audiodescripción en la programación emitida en televisión tradicional 


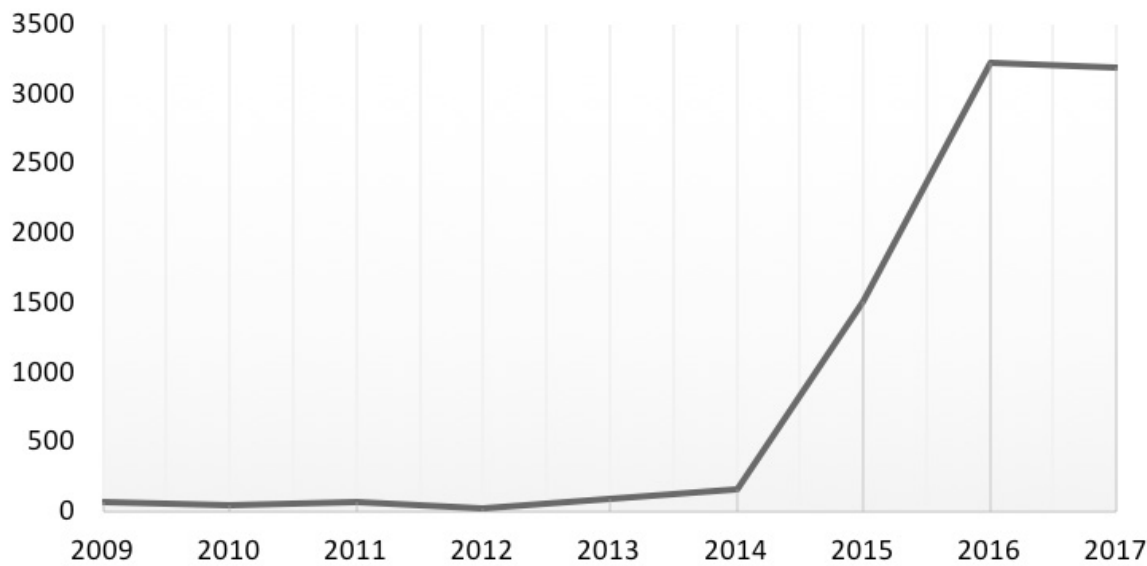

GRÁFICO I. Evolución de la audiodescripción en TVE (horas/año).

FUENTE: elaboración propia a partir de los datos extraídos de RTVE (2008-20I7) ${ }^{\mathrm{I}}$

y online. Para ello, se plantean los siguientes objetivos específicos:

- Analizar y describir la cantidad de programación audiodescrita en TVE en TDT y online, qué tipo de contenidos cuentan con este servicio y en qué horario se emiten en televisión.

- Establecer si la oferta de contenidos audiodescritos en TVE cumple con las cuotas mínimas recogidas en la ley audiovisual.

- Recopilar y mostrar los niveles de satisfacción de las personas ciegas con la audiodescripción de TVE, así como sus principales demandas en relación a este servicio de accesibilidad audiovisual.

\footnotetext{
${ }^{\mathrm{I}}$ Memorias anuales de servicio público y responsabilidad social disponibles en: http://www.rtve.es/rtve/20140609/ transparencia-memorias-servicio-publico/951181.shtml
}

\section{MATERIALES Y MÉTODOS}

En primer lugar, se realizó un análisis de contenido de la programación audiodescrita en TVE durante la temporada 2017-2018, continuando con los últimos datos expuestos en el apartado anterior. Mediante este análisis de contenido, describimos la cantidad de audiodescripción emitida en TVE en el período analizado y determinamos si cumple o no con las cuotas mínimas establecidas por ley.

Concretamente, se analizaron cuatro semanas de programación en televisión y online, ya que los requerimientos de la ley audiovisual para la audiodescripción se establecen por semana. De forma probabilística, se eligieron cuatro semanas distribuidas entre las cuatro temporadas televisivas clásicas (Eastman y Ferguson, 2009) aplicando muestreo aleatorio simple (Cea 
134 D’Ancona, 2012; Igartua, 2006) para dotar al análisis de validez estadística y representativa. Las semanas analizadas fueron: 23-29 de octubre y 25-31 de diciembre de 2017; y 23-29 de abril y 20-26 de agosto de 2018.

En total, se analizó una muestra de 5.044 unidades, cifra superior a las muestras exigidas en un análisis al $99 \%$ de confianza con un margen de error de \pm 2 (Neuendorf, 2002) y que se corresponde con el total de programas de televisión emitidos durante las semanas seleccionadas. De cada una de estas unidades se midió la presencia o no de audiodescripción, el formato televisivo al que correspondía cada programa estudiado y la franja horaria en la que se emitió. Estos tres parámetros se midieron siguiendo las siguientes directrices:

- Para determinar si un programa contaba o no con audiodescripción y calcular los niveles de contenidos audiodescritos, tomamos como referencia la duración de cada programa completo. De este modo se establece la cantidad de programación accesible sobre el total de contenidos emitidos en el período analizado.

- Para determinar el tipo de contenidos audiodescritos, realizamos una clasificación de treinta formatos televisivos distribuidos entre los géneros informativo, educativo y de entretenimiento. Este listado se elaboró tomando como referencia los géneros y formatos propuestos por Creeber (2015) y Gordillo (2009), y se completó con otros formatos propios de TVE hallados durante el análisis. Además, reservamos unas categorías específicas para la programación infantil, puesto que los menores son un colectivo protegido en la legislación audiovisual y la audiodescripción de estos contenidos merece especial atención (Palomo López,
2010). En el apartado de resultados puede consultarse el listado de géneros y formatos televisivos utilizado.

- Por último, para estudiar el horario de emisión de los contenidos con audiodescripción en TDT, se tomó como referencia la división en nueve franjas horarias propuesta por Contreras y Palacio (2001) que se muestra en el apartado de resultados.

Los resultados fueron recogidos en el programa estadístico SPSS 24 y el trabajo fue realizado por un equipo compuesto por cuatro codificadores. La fiabilidad intercodificadores fue de 0.97 según el coeficiente alfa de Krippendorff, aplicado al 10\% del análisis total, superando el 0.80 mínimo exigido (Krippendorff, 2004: 241).

En segundo lugar, realizamos un estudio de recepción mediante encuestas para medir la satisfacción de las personas ciegas que ven TVE con audiodescripción. Para ello, en el cuestionario preguntamos por los mismos parámetros que fueron estudiados en el análisis de contenido. Concretamente, utilizamos preguntas cerradas de respuesta única (dicotómicas y politómicas), y una escala Likert con cinco opciones que van de «muy insatisfecho/a» a «muy satisfecho/a». También se introdujo una pregunta final sobre la satisfacción general con la audiodescripción de TVE en una escala de 0 a 10. Por último, incluimos una pregunta abierta para recopilar posibles carencias y demandas de las personas ciegas no tenidas en cuenta en el cuestionario (Wimmer y Dominick, 2001).

Se realizaron 227 encuestas online a través de la plataforma SurveyMonkey entre enero y marzo de 2018. Para su difusión contamos con el apoyo de la ONCE, que también colaboró en la revisión del propio cuestionario y dio su visto bueno sobre su contenido y forma de ejecución. Solo aquellas personas que respondían afirma- 
tivamente a la pregunta sobre si veían la televisión con audiodescripción accedían al resto del cuestionario para asegurar que quienes respon- dieran a las preguntas fueran usuarios del servicio. A continuación, reproducimos las preguntas del cuestionario:

1. ¿Es usted...?

Hombre

Mujer

Otro (especifique)

2. Por favor, indíquenos su año de nacimiento:

3. ¿Tiene usted alguna discapacidad?

Sí, discapacidad auditiva

Sí, discapacidad visual

Sí, ambas (visual y auditiva)

No tengo ninguna discapacidad

Otra discapacidad (especifique)

4. ¿Utiliza el servicio de audiodescripción para ver la televisión?

Sí

No

5. ¿Cómo de satisfecho/a está con los siguientes aspectos de la audiodescripción de TVE en TDT?

\begin{tabular}{|c|c|c|c|c|c|}
\hline & $\begin{array}{c}\text { Muy } \\
\text { insatisfecho/a }\end{array}$ & $\begin{array}{c}\text { Algo } \\
\text { insatisfecho/a }\end{array}$ & $\begin{array}{c}\text { Ni satisfecho/a ni } \\
\text { insatisfecho/a }\end{array}$ & $\begin{array}{c}\text { Algo } \\
\text { satisfecho/a }\end{array}$ & $\begin{array}{c}\text { Muy } \\
\text { satisfecho/a }\end{array}$ \\
\hline $\begin{array}{c}\text { Cantidad de } \\
\text { programas con } \\
\text { audiodescripción }\end{array}$ & & & & & \\
\hline
\end{tabular}

Variedad de los

programas con

audiodescripción

\section{Horario de los}

programas con

audiodescripción

6. ¿Le gustaría ver los programas de TVE online en directo con audiodescripción? (En en el ordenador, móvil, tablet o smart TV)

Sí

No

7. ¿Le gustaría ver los programas de TVE a la carta con audiodescripción? (En en el ordenador, móvil, tablet o smart TV)

Sí

No

8. En general, ¿cómo calificaría el servicio de audiodescripción de TVE?

\begin{tabular}{llllllllllll}
\hline 0 & 1 & 2 & 3 & 4 & 5 & 6 & 7 & 8 & 10 \\
\hline
\end{tabular}

9. ¿Qué cambios o mejoras introduciría en el servicio de audiodescripción de TVE? 


\section{3. RESULTADOS}

En primer lugar, medimos si la cantidad de audiodescripción emitida en TVE en las temporadas 2017-2018 cumplía con las exigencias mínimas plasmadas en la ley audiovisual española (Ley 7/2010), es decir, 10 horas semanales - lo que equivale a un $5,95 \%$ en canales que emiten 24 horas-. De media, TVE audiodescribió un 7,6\% de su programación en 2017-2018, superando ligeramente el porcentaje mínimo por ley, aunque con diferencias entre las semanas analizadas y los canales.

Por semanas, la audiodescripción de TVE supuso un $6,9 \%$ de su programación en la semana analizada de octubre de 2017, un 7,2\% en diciembre de 2017 , un $8,5 \%$ en abril de 2018 y un $8 \%$ en agosto de 2018. Se aprecia un aumento de más de un punto porcentual entre los niveles existentes al comienzo y al final de nuestro análisis.

Mayor es la diferencia observada entre los canales de la televisión pública. En concreto, La 1, La 2 y Clan superaron, e incluso duplicaron, las cuotas mínimas impuestas por ley, ya que alcanzaron una media de 21,37 horas semanales. En cambio, el canal 24 Horas y Teledeporte no emitieron contenidos audiodescritos, por lo que han sido excluidos del gráfico 2 .

Concretando aún más los resultados por cadenas, La 1, con un 12,2\% de media, audiodescribió de lunes a viernes el programa de recetas Torres en la cocina y las series de sobremesa Serviry proteger y Acacias 38. A estos contenidos se sumaron alguna película semanal y algunos capítulos de programas como Españoles por el mundo, Seguridad Vital, MasterChef Celebrity, etc.

La 2 contó con un 9,5\% de audiodescripción en conjunto. En concreto, contaba con audiodescripción la serie La Pelu-quería en octubre de 2017, pero esta dejó de emitirse durante el resto de las temporadas analizadas, reduciendo los niveles de contenidos audiodescritos. En general, en esta cadena se emitieron algunos documentales y una película semanal con este servicio de accesibilidad.

Por último, en Clan se audiodescribieron las series de animación Yoko, Tutu, El pequeño reino de Ben y Holly, Cuatro amigos y medio y Cleo. También contaba con este servicio el programa Cocina con Clan, que se emitía de madrugada y, en agosto de 2018, se detectó que algunos capítulos del programa Lunnis de leyenda habían empezado a audiodescribirse. La audiodescripción de Clan se completaba con la emisión de uno o dos capítulos diarios de la serie Cuéntame cómo pasó, que no es contenido infantil y que se reponía en horario de madrugada. Resaltamos este aspecto porque implica que más de la mitad de la audiodescripción de Clan no correspondía a programación infantil y, además, se emitió de madrugada, en un horario no accesible para la infancia (del 16,4\% de audiodescripción en la cadena, solo un 7,1\% era contenido para menores).

Asimismo, resulta interesante la distribución de la audiodescripción por día de la semana. Como se ha expuesto, La 1 emite una programación audiodescrita fija de lunes a viernes a la que se suman otros programas en prime time u horario nocturno solo algunos días, lo que da como resultado la diferencia observada en el gráfico 3. Los fines de semana, en cambio, la audiodescripción es similar, y se incluye en programas divulgativos como Seguridad vital y reposiciones de otros contenidos audiodescritos en horario de mañana.

Como se puede observar en el mismo gráfico, la programación audiodescrita de La 2 es más reducida de lunes a jueves, cuando solamente se audiodescriben algunos documentales, y aumenta de viernes a domingo por la suma de las películas del fin de semana. Por el contrario, en Clan, la audiodescripción es bastante similar 


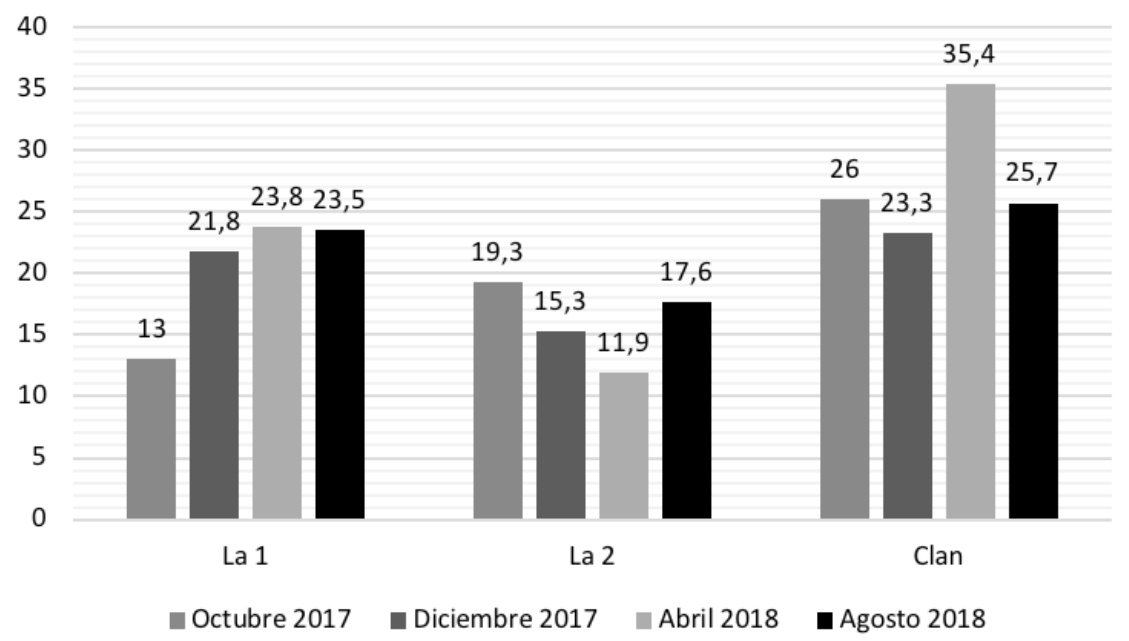

GRÁFICO 2. Audiodescripción en TVE por canal y semana de análisis (horas). FUENTE: elaboración propia

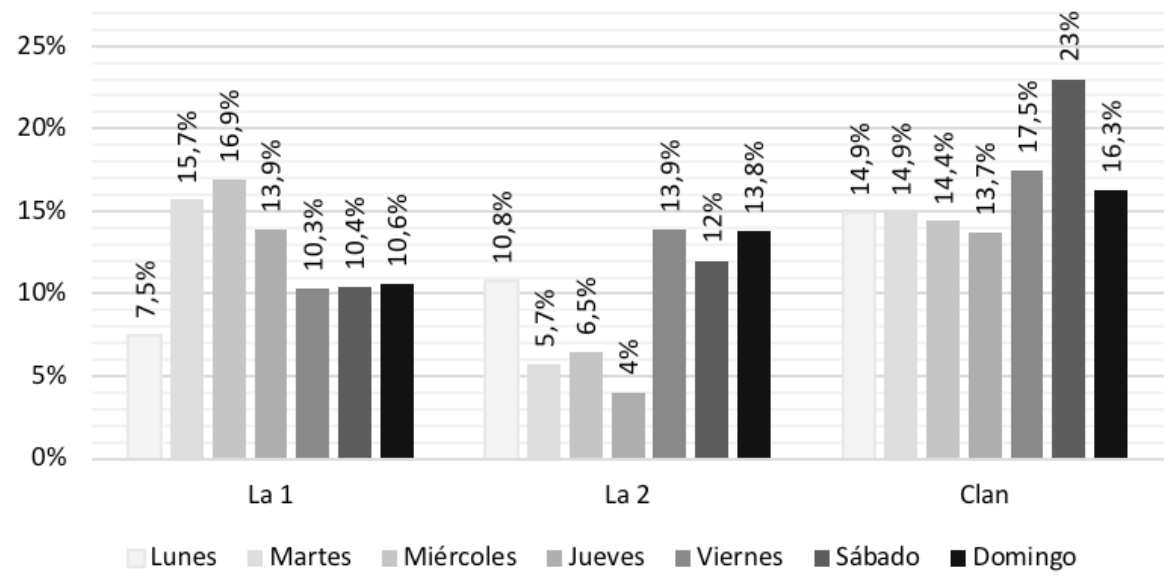

GRÁFICO 3. Audiodescripción en TVE por canaly día de la semana. FUENTE: elaboración propia 


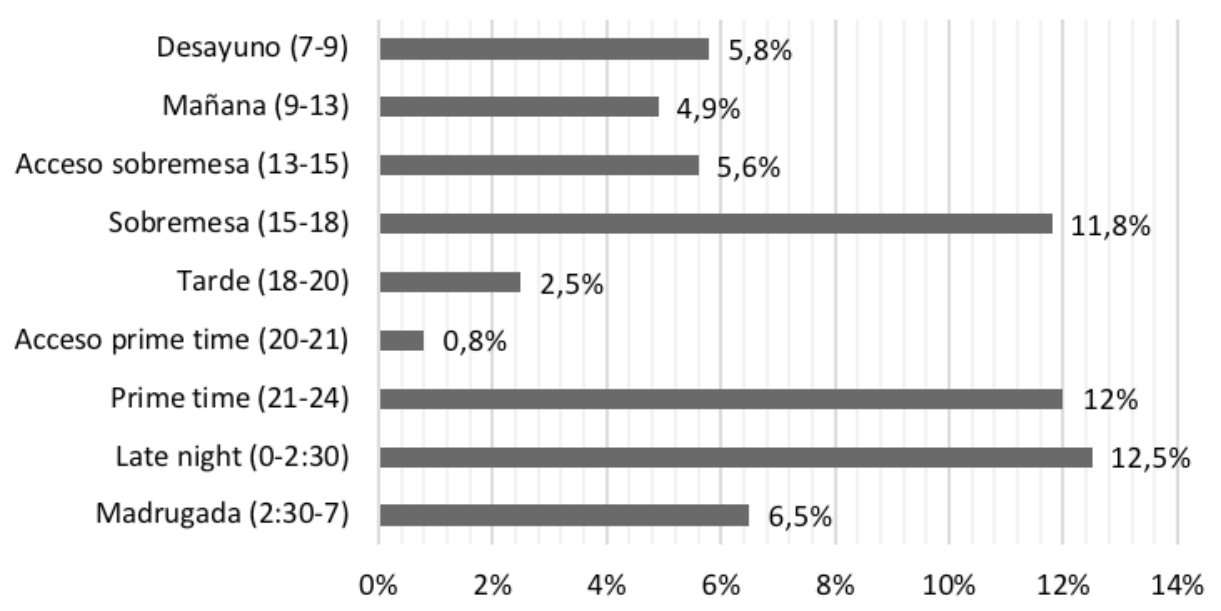

GRÁFICO 4. Audiodescripción en TVE por franja horaria. FUENTE: elaboración propia

todos los días de la semana, puesto que se emiten con audiodescripción las mismas series y en el mismo horario. La excepción es el sábado, cuando aumenta debido a la emisión de la película Antboy con audiodescripción dos veces en el mismo día (el sábado 28 de abril de 2018).

Adentrándonos ahora en la cuestión de las franjas horarias, la ley audiovisual española no establece cuándo deben incluirse los programas con este servicio de accesibilidad, por lo que queda a elección de las televisiones. En TVE, los niveles más altos de audiodescripción se dieron en la sobremesa, cuando se emiten las series Servir y proteger y Acacias 38 en La 1; el prime time, cuando se suelen emitir las películas y otros programas audiodescritos en La 1 y La 2, y el late night, donde se incluyen las reposiciones diarias de la serie Cuéntame cómo pasó en Clan y algunos capítulos audiodescritos del docushow Españoles por el mundo en La 1.

En cuanto al tipo de programas que pueden verse con audiodescripción en TVE, los forma- tos informativos - noticiarios, reportajes, entrevistas, tertulias y magacines informativosprácticamente no contaron con este servicio. En cambio, un $66,8 \%$ de los contenidos de series fueron audiodescritas y un $51,2 \%$ de los talent shows. La siguiente tabla recoge el porcentaje de programación audiodescrita por formato televisivo sobre el total de contenidos de cada formato emitido.

Estos datos hacen referencia al porcentaje de audiodescripción sobre la duración total de los contenidos de cada formato y no sobre el número de programas. Así, por ejemplo, el 10\% del tiempo total de emisión de documentales tuvo audiodescripción, mientras que el $90 \%$ restante no contaba con este servicio.

Además, si agrupamos todos estos formatos en los géneros televisivos a los que corresponden (gráfico 5), observamos que el entretenimiento cuenta con el mayor porcentaje de audiodescripción $(14,7 \%)$, mientras que el nivel más bajo se da en los contenidos informativos $(0,1 \%)$. 
Tabla 1. Audiodescripción en TVE por formato televisivo

\begin{tabular}{|c|c|c|c|}
\hline \multicolumn{2}{|c|}{ Género informativo } & \multicolumn{2}{|c|}{ Género de entretenimiento } \\
\hline Formato & Audiodescripción & Formato & Audiodescripción \\
\hline Noticiario & $0 \%$ & Cine & $12,9 \%$ \\
\hline Noticiario temático & $0 \%$ & Serie & $66,8 \%$ \\
\hline Entrevista & $0 \%$ & Docushow & $29,6 \%$ \\
\hline Debate/Tertulia & $0 \%$ & Reality show & $0 \%$ \\
\hline Reportaje & $1,3 \%$ & Talent show & $51,2 \%$ \\
\hline Magacín informativo & $0 \%$ & Coaching show & $0 \%$ \\
\hline \multicolumn{2}{|c|}{ Género educativo/divulgativo } & Talk show & $0 \%$ \\
\hline Formato & Audiodescripción & Magacín & $0 \%$ \\
\hline Documental & $10 \%$ & Concurso & $9 \%$ \\
\hline Programa cultural & $2 \%$ & Programa de humor & $0 \%$ \\
\hline Programa divulgativo & $7,4 \%$ & Retransmisión deportiva & $0 \%$ \\
\hline \multicolumn{2}{|c|}{ Programación infantil } & Programa de recetas & $22,5 \%$ \\
\hline Formato & Audiodescripción & Galas/Especiales & $0 \%$ \\
\hline Serie de animación & $5,9 \%$ & Otros - entretenimiento & $26,3 \%$ \\
\hline Serie acción real & $0 \%$ & \multicolumn{2}{|l|}{ Otros } \\
\hline Cine de animación & $0 \%$ & Formato & Audiodescripción \\
\hline Cine acción real & $5,8 \%$ & Otros & $0 \%$ \\
\hline Programa infantil & $55,5 \%$ & & \\
\hline
\end{tabular}

Fuente: elaboración propia

Hasta aquí, los resultados que muestran la cantidad de programación audiodescrita, el tipo de programas y el horario en el que se emiten los contenidos con este servicio en TDT. En cuanto a la programación online en emisión simultánea o bajo demanda en la web de RTVE (rtve.es), nuestro análisis muestra que ninguno de los contenidos tuvo audiodescripción durante el estudio. Más tarde, en septiembre de 2018, TVE comenzó a incorporar la audiodescripción en pruebas en el programa Torres en la cocina. Se trataba del mismo sistema que utiliza para incorporar el subtitulado, con un botón en la barra inferior del vídeo en el que se podía activar o desactivar la audiodescripción. Sin embargo, en lugar de extenderse a otros contenidos, esta opción fue eliminada y sigue sin estar disponible.

Por otro lado, como avanzamos en el apartado metodológico, encuestamos a 227 personas ciegas usuarias a quienes preguntamos por su grado de satisfacción con los parámetros estudiados en nuestro análisis y obtuvimos los siguientes resultados. 


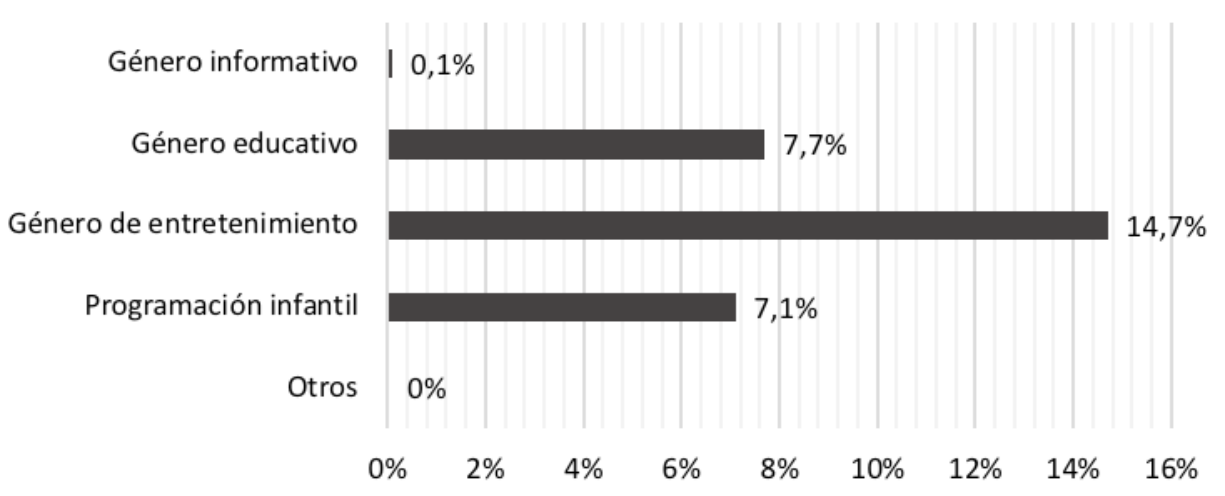

GRÁFICO 5. Audiodescripción en TVE por franja horaria. FUENTE: elaboración propia

Cantidad de programas con audiodescripción

Variedad de los programas con audiodescripción

Horario de los programas con audiodescripción

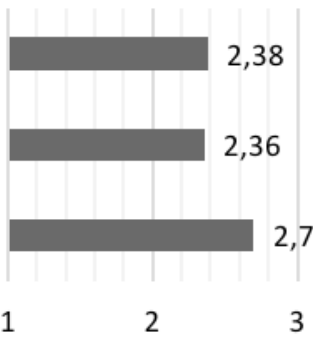

3

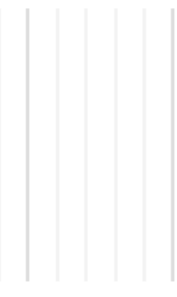

4
5

GRÁFICO 6. Promedio de satisfacción con siguientes aspectos de la audiodescripción de TVE. FUENTE: elaboración propia

Convertimos las cinco opciones de la escala Likert utilizada — de «muy insatisfecho/a» a «muy satisfecho/a»- a valores numéricos del 1 al 5 y observamos que ninguna de las variables estudiadas llegaba al 3, que sería la puntuación intermedia y supondría el aprobado (gráfico 6). De hecho, en las dos primeras variables, las opciones más escogidas fueron las más bajas («muy insatisfecho/a» $\mathrm{y}$ «algo insatisfecho/a»). Estas valoraciones encajan con el hecho de que la demanda más repetida al final de la encuesta fuera que se aumentase el número de programas con audiodescripción. Asimismo, recogimos más de una decena de respuestas que pedían más diversidad de contenidos audiodescritos y mayor variedad en los horarios.

En nuestra encuesta, preguntamos a las personas usuarias de este servicio en televisión si querrían tenerlo disponible también en los contenidos en línea. De las 220 respuestas recopiladas, un $95,45 \%$ fueron afirmativas para la emisión online en directo y un $97,73 \%$ para los contenidos a la carta. Según estos datos, una abrumadora mayoría de las personas encuestadas querrían ver estos contenidos online con audiodescripción, ya sea en la emisión simultánea o bajo demanda. 
Finalmente, pedimos a las personas encuestadas que valorasen la audiodescripción de TVE en una escala de 0 a 10 . En esta valoración general, las encuestas le otorgaron una media de 6,34. De hecho, la calificación que las personas encuestadas marcaron en más ocasiones fue el 6, mientras que la menos seleccionada fue el 10. Esta cuestión muestra cómo la audiodescripción de TVE aprueba en nuestra encuesta, pero tiene un claro margen de mejora para los usuarios.

Por ello, la última pregunta del cuestionario era una pregunta abierta en la que se pedía a las personas ciegas que ven contenidos de TVE con audiodescripción que compartiesen aquellos cambios o mejoras que introducirían en este servicio. Se trataba de recopilar y exponer las prioridades de los propios usuarios y fue respondida por 168 personas. De ellas, más de 60 personas solicitaron un aumento de los programas con audiodescripción, siendo esta la respuesta que más veces se repite. Asimismo, hasta 16 personas solicitaron más diversidad en los formatos de los programas con audiodescripción y varias pidieron también mayor variedad en los horarios.

Tras esta cuestión estaría la necesidad de incorporar este servicio a la emisión online y a la carta, argumento que se repitió en más de 30 ocasiones. Recuperamos aquí la respuesta literal de una persona usuaria sobre la importancia de que estos contenidos sean audiodescritos: «En el siglo XXI, no es que a mí o a otros nos guste ver a la carta y online los contenidos audiodescritos; es que resulta obligatorio para no discriminar a nadie. Y no hay excusas para que esto no se lleve a cabo».

Además, las demandas recogidas en nuestras encuestas abarcaban otros aspectos no analizados en este estudio, pero que demostraron ser relevantes para los usuarios. Por ejemplo, las personas encuestadas expusieron su dificultad para saber con antelación qué programas van a emitirse con audiodescripción y reclamaron hasta en 13 ocasiones que se aporte esta información previamente.

Sobre esta cuestión, los programas con audiodescripción en TVE cuentan con el símbolo visual «AD» que aparece junto al de subtitulado o lengua de signos al principio del programa. Para conocer esta información con antelación es necesario recurrir al teletexto de TVE o a la guía de programación (EPG). No obstante, la información del teletexto no es completa (no aparecen las series audiodescritas de Clan, por ejemplo) y la de la EPG varía de un televisor a otro (esta cuestión fue corroborada por todos los codificadores de este estudio en la preparación de nuestro análisis). Por último, el blog versionaccesible.blog difunde la previsión de programación audiodescrita de TVE, pero no la de las televisiones privadas o autonómicas.

Para terminar este apartado, en nuestra encuesta también recopilamos demandas relacionadas con la calidad del servicio. En esta línea, hasta 27 personas dijeron que el volumen de la audiodescripción no se correspondía con el del programa, dificultando la recepción. Para ello, los propios usuarios nos propusieron soluciones como la siguiente: «Que la audiodescripción se emita en una pista conjunta con el audio del programa, y no por separado». El otro aspecto cualitativo que también aparece más de una decena de veces es que la audiodescripción, en ocasiones, no está sincronizada con el programa y se producen solapamientos entre el servicio de accesibilidad y los diálogos. Aumentar la variedad y expresividad de las voces; eliminar elementos redundantes y ampliar la descripción de aspectos relevantes; incluir información sobre los actores que representan a los personajes en series y películas; narrar la información escrita en pantalla; hacer descripciones objetivas y que se tengan en cuenta las sugerencias de la audiencia completan las 
142 aportaciones sobre aspectos cualitativos o relacionados con la calidad del servicio.

\section{DISCUSIÓN Y CONCLUSIONES}

En primer lugar, para contextualizar los resultados, la audiodescripción comenzó a crecer en TVE en 2015 y en 2016 alcanzó un 7,3\%, cifra muy similar al 7,6\% registrado en nuestro análisis en las temporadas 2017-2018. Este porcentaje equivale a una media de 21,37 horas semanales en cada uno de los canales que cuentan con este servicio - La 1, La 2 y Clan-. De este modo, las cadenas con audiodescripción no solo cumplen con la cuota mínima de 10 horas semanales dictada por la ley audiovisual española (Ley 7/2010), sino que llegan a doblar la cifra. Sin embargo, las personas usuarias encuestadas valoran la cantidad de audiodescripción en TVE con un 2,38 sobre 5 , lo que indica un claro margen de mejora según sus niveles de satisfacción. Además, ni el canal 24 Horas ni Teledeporte ofrecían este servicio, aunque la ley no hace ninguna distinción sobre canales.

La audiodescripción se concentra en programas grabados, destacando en los formatos de ficción - cine y series-, algunos programas de entretenimiento, como talent shows o programas de recetas, y documentales. Es cierto que los programas en directo, que son principalmente informativos, no son susceptibles de incorporar este servicio porque es necesario visionar el programa con antelación para preparar la audiodescripción. Sin embargo, sí consideramos interesante la incorporación de una guía de buenas prácticas para que los presentadores incluyan la información visual que sea relevante para el seguimiento del programa en su propio discurso. Por ejemplo, en el caso de los informativos se deben tener en cuenta cuestiones como las declaraciones en otros idiomas, que suelen estar subtituladas en español, pero no audiodescritas. La introducción de audiosubtítulos en estos casos solucionaría el problema y garantizaría la accesibilidad para las personas ciegas.

Además, TVE no contó con audiodescripción en sus contenidos online en directo ni a la carta durante el período analizado, a pesar de que el consumo audiovisual a petición sigue creciendo ampliamente en España (CNMC, 2019). Esta es una cuestión muy demandada por las personas encuestadas. Una vez concluido nuestro análisis, TVE introdujo la audiodescripción a la carta en pruebas con un sistema similar al del subtitulado, es decir, incorporando un botón en la barra del vídeo para activarla o desactivarla. Sin embargo, este servicio ha desaparecido en la web de RTVE de momento.

Nuestras encuestas recopilaron también otras demandas que van más allá de los aspectos estudiados en el análisis. Por ejemplo, recibimos múltiples respuestas que hablaban de la dificultad para encontrar programas audiodescritos, tanto en televisión tradicional como a la carta. Asimismo, en la preparación de nuestro análisis pudimos comprobar que la programación publicada por TVE en su web no cuenta con este dato y que la guía de programación muestra claras diferencias de un televisor a otro. Estas cuestiones dificultan saber de antemano qué programas están audiodescritos y deben ser solucionadas para que las personas con discapacidad visual puedan disfrutar de los contenidos accesibles.

Para concluir, conectamos nuestros resultados con las premisas sobre la vinculación entre la accesibilidad y la inclusión social presentadas al comienzo. En nuestras encuestas pudimos comprobar cómo las personas usuarias coinciden en esta idea con otros autores citados (Jiménez Hurtado y Soler Gallego, 2015; Rimmerman, 2012) y con lo expuesto en la normativa actual, y corroboran la importancia de la accesibilidad 
universal a la televisión para garantizar la inclusión social. Así se demuestra en algunas de las respuestas extraídas en el apartado de resultados y en las exigencias de mayores niveles de audiodescripción. Del mismo modo, es necesario recordar que el concepto de pluralismo mediático que representa e incluye a cada mayoría y a cada minoría social y cultural (Comisión Europea, 2009) comprende también la audiodescripción para promover la accesibilidad universal y, a su vez, la inclusión social. Todas estas cuestiones cobran aún más importancia en la televisión pública, que tiene entre sus funciones la accesibilidad universal y el respeto y la atención a la diversidad y a las minorías (Blumler, 1993; Medina y Ojer, 2009; Michalis, 2010).

Los datos hallados en esta investigación abren una vía para futuros trabajos de investigación sobre la calidad de la audiodescripción desde el punto de vista de los usuarios. Para próximas investigaciones sería interesante indagar sobre las demandas expuestas en las encuestas a través de métodos cualitativos como entrevistas en profundidad ofocus group, o diseños experimentales para determinar si la calidad de la audiodescripción permite la comprensión completa de los contenidos televisivos.

\section{REFERENCIAS}

BACHMEIER, Cristina (2014): «Barrier-free Access to audiovisual content. A fundamental human right», en Susanne Nikoltchev (ed.) Enabling Access to the Media for All. IRIS plus 2014-3, Strasbourg: European Audiovisual Observatory, 7-22.

BENECKE, Bernd (2004): «Audio-Description», META, 49/1, 78-80.

Blumler, Jay G. (ed.) (1993): Televisión e interés público, Barcelona: Bosch.

CMT (2010): Informe de accesibilidad en los servicios televisivos, https://docplayer.es/11825160-Informe- de-accesibilidad-en-los-servicios-televisivos-diciembre-2010-cmt.html

CNMC (2019): Panel Hogares CNMC: Los españoles llaman desde el móvily (queman) el WhatsApp, https:// blog.cnmc.es/2019/10/31/los-espanoles-llamandesde-el-movil-y-queman-el-whatsapp-panelhogares-cnmc/

Comisión Europea (2009): Independent Study on Indicators for Media Pluralism in the Member States - Towards a Risk-Based Approach, http://ec.europa.eu/ information_society/media_taskforce/doc/pluralism/pfr_report.pdf

Contreras, José Miguel y Manuel Palacio (2001): La programación de televisión, Madrid: Sintesis.

CreEber, Glen (2015): The Television Genre Book, London: BFI.

Directiva 2010/13/UE del Parlamento Europeo y del Consejo, de 10 de marzo de 2010, sobre la coordinación de determinadas disposiciones legales, reglamentarias y administrativas de los Estados miembros relativas a la prestación de servicios de comunicación audiovisual, https://eur-lex.europa. eu/legal-content/ES/TXT/PDF/?uri=CELEX:32010 L0013\&from $=E S$

Eastman, Susan y Douglas Ferguson (2009): Media Programming: Strategies and Practices, Boston: Thomson/Wadsworth.

Franco, Eliana, Deise Medina Silveira y Bárbara dos SANtos Carneiro (2015): «Audio Describing for an Audience with Learning Disabilities in Brazil: A Pilot Study», en Rocío BAÑos y Jorge Díaz-CinTas (eds.) Audiovisual translation in a global context. Mapping and ever-changing landscape, Basingstoke: Palgrave Macmillan, 99-109.

FrYer, Louise (2016): An Introduction to Audio Description. A Practical Guide, London: Routledge.

Gordillo, Inmaculada (2009): La hipertelevision: géneros y formatos. Quito: Quipus.

Igartua, Juan José (2006): Métodos cuantitativos de investigación en comunicación, Barcelona: Bosch.

Jiménez Hurtado, Catalina y Silvia Soler Gallego (2015): «Museum Accessibility through Translation: A corpus Study of Pictorial Audio Description», en Jorge Díaz-Cintas y Josélia Neves (eds.) Audiovisual 
144 Translation. Taking Stock, Newcastle-Upon-Tyne: Cambridge Scholars Publishing, 277-298.

KrIPPEndorfF, Klaus (2004). Content Analysis: An Introduction to Its Methodology, Thousand Oaks (Calif.): Sage.

Kurz, Ingrid y Brigitta Miкulasek (2004): «Television as a Source of Information for the Deaf and Hearing Impaired. Captions and Sign Language on Austrian Television», META, 49/1, 81-88, doi: 10.7202/009023ar.

Labio Bernal, Aurora (2014): «El eterno debate sobre la concentración mediática en la Unión Europea. Del plan Reding-Wallström a la Iniciativa Ciudadana por el Pluralismo», en Manuel CHAPARro (ed.) Medios de proximidad: Participación social y politicas públicas, Málaga: Luces de Gálibo, 55-72.

Lopez, Mariana, Gavin KeARney y Krisztián HofstäDter (2018): «Audio Description in the UK: What works, what doesn't, and understanding the need for personalising access», British Journal of Visual Impairment, doi: 10.1177/0264619618794750.

McDaniel, Carl y Roger Gates (2005): Investigación de mercados, México D.F.: Thomson.

Medina, Mercedes y Teresa OJer (2009): «Valoración del servicio público de televisión. Comparación entre la BBC y TVE», Revista Latina de Comunicación Social, 12/64, 275-299, http://www.revistalatinacs.org/09/art/24_823_42_ULEPICC_11/ Medina_y_Ojer.html

Michalis, Maria (2010): «EU Broadcasting Governance and PSB: Between a Rock and a Hard Place», en Petros Iosifidis (ed.) Reinventing Public Service Communication: European broadcasters and beyond, Basingstoke: Palgrave Macmillan, 36-47.

Molina SAORín, Jesús (2017): La discapacidad empieza en tu mirada: las situaciones de discriminación por motivo de diversidad funcional: escenario jurídico, socialy educativo, Madrid: Delta.

Neuendorf, Kimberly A. (2002): The Content Analysis Guidebook, Thousand Oaks: Sage.

ONU (2006): Convención sobre los derechos de las personas con discapacidad, http://www.un.org/esa/socdev/enable/documents/tccconvs.pdf

Palomo López, Alicia (2010): «The benefits of audio description for blind children», en Jorge DíAz-CIN- tas, Ana Matamala y Josélia Neves (eds.) New insights into audiovisual translation and media accessibility: Media for All 2, Amsterdam: Rodopi, 213-226.

Rimmerman, Arie (2012): Social Inclusion of People with Disabilities. National and International Perspectives, Cambridge: Cambridge University Press.

SNYDER, Joel (2005): «Audio description. The visual made verbal», en Jorge Díaz-Cintas (ed.) The didactics of audiovisual translation, Amsterdam: John Benjamins, 191-198.

Storch de Gracia y Asensio, José Gabriel (2007): «Construcción jurídica del derecho a una televisión accesible», TRANS: Revista de Traductología, 11, 115-134, doi: 10.24310/TRANS.2007.v0i11.3101.

VerCAuteren, Gert (2007): «Towards a European guideline for audio description», en Jorge Díaz-CinTas, Pilar Orero y Aline Remael (eds.) Media for All. Subtitling for the Deaf, Audio Description, and Sign Language, Amsterdam: Rodopi, 139-149.

WiMmER, Roger y Joseph Dominick (2001): Introducción a la investigación en medios masivos de comunicación. México: International Thomson. 